\title{
Drug Addiction: Therapeutic Communities Breaking the Cycle of Addicted Mothers
}

\author{
$\mathrm{J}-\mathrm{F}$ \\ Correspondence: Dr J-F, Lecturer in Human Development, University of Tasmania, Locked Bag 1307, \\ Launceston TAS 7250, Australia
}

Received: October 24, 2013 Accepted: November 13, 2013 Available online: December 11, 2013

doi:10.11114/ijsss.v2i1.251

URL: http://dx.doi.org/10.11114/ijsss.v2i1.251

\begin{abstract}
Fresh Hope (FH) is an in-house residential therapeutic drug-rehabilitation facility assisting mothers with children to break the cycle of addiction. FH uses 'community' as democratic method to address: drug addiction, anti-social behaviors, re-establish positive relationships with children and to live drug free. Residents take part in a 4 stage 18 months Community Drug Rehabilitative Treatment Experience/Program and a semi-structured Personal/Community Engagement Experience/Program. An archival study design was used to measure the efficacy of program against two measures: completion of stages and whether residents were living drug-free after successfully graduating from the program. Since 2000 to 2011 around 144 participants entered the program. Over $49 \%$ successfully completed Stage 1 (Detoxification Phase); with 25\% completing Stage 2, and 15\% ( $\mathrm{n}=$ 21) graduating from the full program (Stage 3 and 4). A post-release follow up of graduates $(n=21)$ after 11 years found that $90 \%$ are living drug-free (ranging from 1 to 12 years) and are productive members in the community. The value in this finding is that therapeutic communities operating under the premise of a democratic method of treatment provide a useful non-instructive alternative to clinical treatments.
\end{abstract}

Keywords: drug addiction, therapeutic communities, mothers, drugs, rehabilitation

\section{Introduction}

\subsection{Overview of Fresh Hope}

Fresh Hope $(\mathrm{FH})$ is an in-house non-medical, non-pharmacological residential drug rehabilitative therapeutic facility for mothers (i.e., drug dependent/substance abusers) and their children (Fresh Hope, 2005). Fresh Hope has two communal houses. Both are large Queenslander houses with a capacity for nine mothers and up to twenty children at any given time. FH has been operating since 1999 and is located in Toowoomba, Queensland. FH accepts children along with the mothers, wherein it provides support/treatment for both mothers (e.g. addiction) and children (e.g. trauma \& attachment). In line with De Leon (1986), Nielsen and Scarpitti (1997) FH is a “... therapeutic community ... a total treatment environment ... a residential 24-hour-per day learning experience in which drug user's...[i.e. drug dependent]... transform ... [their] conduct, attitudes, values, and [positive].... emotions are introduced monitored, and mutually reinforced as part of the daily routine..." (p, 279; Clinton, 1996; De Loen, \& Jainchill, 1982; De Leon, Sacks, Staines, \& McKendrick, 2000; Kennard 2004; Vaughan, 2004; Ward, 2003; Wolfman 2009). The criterion to enter into FH is any mother wanting to live drug free irrespective of the severity of the drug/substance and/or dependency/abuse (Cardoso, Chan, Berven, \& Thomas, 2003).

Community in itself is seen as "treatment" in addressing drug addiction. FH uses the seminal works of Jones (1957) and defines its therapeutic community in terms of democratic method, "... a group of people assembled for the purpose of healing..."; whereby "institutional resources" such as staff, clients, professionals and peers are "... self-consciously' pooled together for the purposes of treatment ..." (p, 200; Kennard, 2004; Mills \& Harrison, 2007; Smith, 2011). Informed by Judea-Christian ethos in its service model FH is underpinned and informed by principles of therapeutic community as a family home similar to that of Synanon in California USA and that of the Henderson Hospital (Vaughan, 2004). Fresh Hope uses its community (mothers, ex-graduates, administrative staff, clinicians, support staff, children, volunteers and community members) as democratic method of treatment to address: addiction, anti-social behaviors, residents (i.e. mothers) to re-establish positive relationships with their children; and for residents to become productive members in the community.. The staff of FH are divided into two categories. Category 1: Administrative staff (e.g., Business Manager) and Category 2: 
Support Staff (Senior Counselor, GP, Medically Trained Counselor, Graduates, Volunteer community support staff, House Parents \& Case Workers). House Parents (i.e. half Graduates and half from the community) and Case Workers (i.e., some are Graduates and others are employed) oversee dailychores/errands necessary for residents to complete for the smooth running of the facility (Kennard, 2004). At least two staff families live on site to not only aid in modeling positive and supportive behaviors, but to add to the therapeutic culture of support and care (Smith, 2011). All staff model altruistic behaviors of fairness, kindness, integrity and importantly delivers unequivocal support toward recovery (Provert, 2006).

The structure of FH is similar to that of Sober Living Houses (SLHs) where residents pay fees (e.g., for accommodation, food and professional services) and can stay as long as they wish by continuing to make positive change and by becoming productive members in the community (Polcin \& Henderson, 2008). Also, similar to that of SLHs the various support programs and activities within FH are also not monitored by a licensed body or an agency. However, FH adheres to all legislative policies in terms of mandatory reporting, work place health and safety; and models a philosophy of recovery and sustainable change in its day to day operations (Fresh Hope, 2005). Unlike SLHs, FH uses a specialist, such as: General Physicians for regular medical check-ups and a Medically Trained Counselor to complete a comprehensive psychiatric assessment on residents in terms of co-morbid and/or mental health concerns (Fresh Hope, 2005).

At the time of admission into FH all residents undergo an informal (i.e. semi-structured interview with the Senior Counselor) assessment in terms of: readiness, suitability and motivation toward treatment (Kaplan \& Broekaert, 2003). At induction residents are briefed on the therapeutic community experience and all residents make a contract agreement to fully participate in the program and give consent to documenting treatment progress and their contributions to FH and the wider the community. To ensure the integrity of the program, all residents consent to random drug and alcohol urine test during their stay in the facility. FH delivers a unique service paradigm as it customizes its support services to suit mothers and children and consequently incorporates a family culture by housing mothers and children together; using colloquial extended family language (e.g. children refer to other mothers as aunties... etc.); providing a day care and afterschool program for children and their mothers; using a gender-specific curriculum program to teach parenting skills to mothers; a daily program for children and a parenting program for mothers to develop their own parental responsibilities (Fresh Hope, 2005; Sacks et al., 2004). Over the eighteen month two separate therapeutic experiences are offered: one for mothers and one for children. In particular the mothers' therapeutic experiences are further divided into two distinct programs: one, a structured non-clinically Community Drug Rehabilitative Treatment Experience/Program, which is divided into four stages; and two, a semi-structured Personal/Community Engagement Experience/Program (Fresh Hope, 2005; Polcin \& Henderson, 2008).

\subsection{Review of the Literature}

The National Drug Strategy 2010-2015 (NDS) using Collins and Lapsley (2008) work, note that licit and illicit drug use incur a great economic, social and medical cost, which is around $\$ 56.1$ billion in 2004/5, over and above personal and psychological loss and trauma which are difficult to quantify. Currently there are a number of strategic State and Federal policies, programs and services that address licit and illicit drug dependency/abuse problems in Australia (National Drug Strategy, 2010-2015; National Drug Strategy Household Survey, 2011). And while conventional treatments are relevant and applicable in treating addictions, therapeutic communities also seem to provide a unique non-medical and non-pharmacological approach to drug-rehabilitation (De Leon, 1993; Mills \& Harrison, 2007; NDS; Provert, 2006; Vaughan, 2004; Wolfman, 2009; Zweben, 1993). Therapeutic Communities (TC) are argued to provide a necessary and important way to address drug abuse and addiction as they profess to offer a more realistic context (i.e. community setting) for rehabilitation and sustainable change (De Leon, 1989; De Leon, 2000; Lees, Manning \& Rawlings, 2004; Nielsen \& Scarpitti, 1997; Polcin \& Henderson, 2008; Sacks et al., 2004). Given the non-clinical nature, community therapeutic experiences are commonly criticized to be merely social rehabilitation rather than to address the core cause of the pathology (Wolfman, 2009).

FH uses socio-psychological approach in explaining how behaviors are acquired (Gottdiener, 2001; Huffman, 2004; Mills \& Harrison, 2007; NIDA, 2004). In line with the seminal work of Sutherland (1947), FH understands that negative, maladaptive, anti-social and offending behaviors primarily happen in association with others; that they are learnt (e.g. either by vicarious reinforcement and/or direct positive reinforcement) and seem to have specific motivation/s; that individuals often exercise differential associations with little or no sense of conformity; and that these individuals are likely to have early negative experiences compounded with several other risk factors - which all collectively seem to contribute toward individuals engaging and practicing maladaptive behaviors such as drug taking/addiction (Andrews and Bonta, 1994; Gottfredon and Hirschi, 1986; 
Yates \& Wilson, 2001). Based on Sutherland's (1947) view, FH further argues that those individuals who are addicted to substances actively engage in unhealthy/maladaptive behaviors (i.e. consuming drugs) in association with others and within environments that promote, support and sustain behaviors as the norm, in other words there appears to be a triadic interaction across the three Social Cognitive Theory (SCT) constructs: individual, behavior and environment (Bandura, 1986). SCT recognizes that personal experiences/characteristics (e.g. specific motivations, loss, abuse and/or, trauma) can lead to the acquisition of and/or learning of maladaptive behaviors as coping mechanisms and/or as means to managing/surviving the anguish of negative experiences, and that particular environments and contexts maintain and sustain such behaviors. In this SCT explains that behavior, personal attributes and environment are both independent and inter-dependent constructs that collectively come to influence, motivate and sustain behaviors. SCT argues that for a positive change in behavior to occur all of these constructs must be addressed (Bandura, 1986). For SCT maintains that behavior, self-development, adaptation to change are facilitated through triadic reciprocal interactions between personal characteristics, behavior and environment and that all these constructs must be addressed to influence an individual's development (e.g., develop positive behavior) (Bandura, 1986, 2003, J-F, 2011).

In line with the above, FH uses various constructs (e.g. behavior, personal attributes and environment) of SCT to ensure that therapeutic and recovery support services not only address drug addiction and anti-social behaviors but also foster positive self-identity, learn positive behaviors and for mothers to re-establish positive relationships with their own children within a safe environment (Bandura, 1986). In other words, if we want to foster positive behaviors: at an individual level, they must be taught; at a behavior level, they should be modeled and/or practiced; and, that a supportive environment is necessary to foster development of these positive behaviors, hence all three constructs must be taken into account to influence and shape behavior. The Community Drug Rehabilitative Treatment Experience/Program while self-developed by FH is structured and informed by a Triadic Interaction Model of Social Cognitive Theory (SCT) (Bandura, 1986).

The Community Drug Rehabilitative Treatment Experience/Program: Primarily aims to assist in breaking the cycle of addiction, anti-social and to live drug free; and secondly, it aims for residents to develop positive relationships with their own children, family and the community. This program is divided into two parts: one, residents attend weekly one-on-one Drug Education Counseling sessions with the Senior Counselor; and two, a structured four stage 18 month treatment program. The four stages are: detoxification/orientation (6weeks), developing positive relationships (18weeks), transitional/independent living (24weeks) and community integration (24 weeks); to this end even though this program is informed by several works, it primarily draws from the works of Sacks et al (1997). The duration of the program is in line with the De Leon (1993) who argues that a successful therapeutic community experience requires at least eighteen months to twenty four months of active participation. To this end, Toumbrourou, Hamilton and Fallon (1998) found that “... time spent in treatment ....through levels of treatment progress... has been the most commonly reported variable and has been found to be the most predictor of success following treatment..." (p. 1051-1052). However, Polcin and Henderson (2008) found that after a six month period about 56\% ( $n=130)$ had left the rehabilitation program. Wolfman (2009) also notes that low rates of treatment retention appear to be common in drug treatment given the complexity surrounding addictions and the various factors that influence behavior. Equally, given the nature and the process and/or progress of treatment within therapeutic community, some might argue that there is no quantifiable evidence of treatment at the each stage of the program (Perfas \& Spross, 2007).

Stage 1: Detoxification/Orientation. As part of the preliminary assessment each resident has an interview with a Senior Counselor, and they complete a self-assessment and are assigned a Case-Worker. The purpose of this stage is total and complete detoxification from all illicit drugs and to address victim thinking (Zweben, 1993).

All residents (i.e. mothers) go through an induction and are given a list of tasks/chores they need to complete each day and are allocated a bed room in the communal house. During this stage residents are not allowed to or any contact with anyone one outside the facility, except for written letters which are supervised. However, children in the facility are allowed phone calls and can contact others (e.g. grandparents or fathers) outside the facility.

Stage 2: Developing Positive Relationships. Each resident undergoes a full Psychiatric assessment in terms of their drug history and mental health. This assessment assists Case Workers to implement the needed support for residents. During this stage residents attend several structured programs such as: Parenting Workshop, Anger Management, Positive Lifestyle Program, Horses for Healing, Communication skills, Migration of Identity Map, and they are also given several opportunities to develop intrapersonal, communication skills and interpersonal skills. Importantly, during this stage residents begin to work through re-establishing positive relationship with their own children.

Stage 3: Transitional/Independent Living: Each resident attends an in-house re-lapse prevention program. During 
this stage, residents are given a leadership position to mentor, supervise and support residents in Stage 1 and Stage 2. Further, they are also given responsibility to oversee a few community building programs, such as: maintaining gardens and organizing events for children. It is at this stage that residents begin to seek and make contact with support agencies outside of the facility, attend job interviews, set up positive life plans and go through an in-house financial management program. Importantly, during this stage residents begin to work through re-establishing positive relationships with their family and the wider community.

Stage 4: Community-Integration: After graduation from Stage 3, residents move out of the facility and start to live in independent housing (e.g. rent or lease) and either begin employment or training. However, all residents continue to: attend (at least one) in-house weekly sessions in the facility; have regular meetings with their Case-Workers and the Senior Counselor; participate in community events; and are, encouraged to attend AA and NA groups in the community. Further, residents begin to take an active part in the wider community as they begin to live productively through employment/training and by volunteering in churches and organizations. Participation in each of these stages takes on a linear mode such that after successful completion of each stage, residents graduate to the next stage. If breaches in behaviors occur at any given stage residents are placed back in stage one (i.e. for a period of time subject to review of behavior) irrespective of the stage they have graduated to or from.

At an individual level: The purpose of the four stages of Community Drug Rehabilitative Treatment Experience/Program is very much to re-teach, re-educate and address maladaptive behavior, motivations and work through the process of living and sustaining a drug-free life (Safran \& Muran 1998). FH recognizes that there are specific motivations that lead individuals to unhealthy and/or maladaptive behaviors and that these behaviors are learnt and expressed (i.e. acted out) in association with others. $\mathrm{FH}$ argues that a positive sense of self, progressive constructive core beliefs, pro-social conventional norms/ values and productive skills are necessary for one to begin their drug-free life and that these need to be explicitly re-taught to individuals who are substance abusers (Fresh Hope, 2005). Such process is likely to empower individuals to not only address their addictive like behaviors but provide an opportunity to positively blossom like a flower (Kennard, 2004).

At an environment level: FH contends that over and above the individual's struggles (i.e. pathology of addiction), it is very likely that these anti-social communities compound and reinforce addictive behaviors to be customary, normal and not ruinous. Wolfman (2009) argues that substance use and abuse is a social phenomenon and Murray et al. (2007) supporting this assertion notes that such social factors are not only criminogenic risk factors but that they can also directly lead to and maintain anti-social behaviors, as drug cultures (i.e. environment) are very likely to disregard any and all adherence to social conventions, norms or even laws, and that the governance around anti-social communities are likely to make it very difficult for individuals to break free from such behaviors (Andrews \& Bonta, 1994; Gottfredon \& Hirschi, 1990). In this, FH primarily offers a safe, protective and drug-free environment and positions "... individuals to overcome social pressure ... [to engage in drugs/alcohol] ..." by providing a drug free culture (Polcin \& Henderson 2008, p. 154).

At a behavior level: FH distinguishes itself to be purposeful and constructive and offers mothers who are drug dependent/substance abusers a meaningful opportunity to learn positive behaviors and skills. FH creates several opportunities for residents to develop positive relationships with others and to regain a sense of productive identity. In this, FH takes a unique and pragmatic approach of primarily starting with re-establishing positive relationships between mothers and their children as fundamental to developing positive behaviors. Having worked through establishing positive relationships with the children, the residents then begin to work "... through a variety of social-psychological mechanisms ... [for example]... social modeling/learning [reciprocal learning], help giving [behaviors]... [positive] ... peer influence... and ... [learning positive] ... social norms ... [is likely to]... change attitudes and behaviors ...", such that new and positive behaviors can be taught and learnt (Wolfman, 2009, p.7). Thus, FH models positive therapeutic behaviors (e.g. sense of belonging, secure attachment and altruistic behaviors) by fostering spontaneous interactions (e.g. between residents, peers, children and staff) to create a positive and beneficial culture of optimism and construct based on a philosophy of family and propitiousness which is much needed to break the cycle of addiction (Jones, 1957).

The focus of Personal/Community Engagement Experience/Program is primarily around building a supportive community around the resident. Through the Personal/Community Engagement Experience/Program, FH practices: democratization, reality confrontation and altruistic communalism to create a positive pro-social therapeutic community (Fresh Hope, 2005; Kaplan \& Broekaert, 2003; Lees et al., 2004; Rapoport, 1960). The background to the development of this program is primarily from the seminal works of De Leon (2000) in terms of allowing residents to experience a sense of community and belonging through various aspects of the community (i.e. language, culture... etc.). In line, a number of structured and unstructured activities (e.g. personal, spiritual, social, training and employment) are made available to residents so that they can experience a 
positive pro-social community through social inclusion exercises, positive participation in groups and through community integration workshops which are deemed to be necessary for a full recovery (Fresh Hope, 2005; Kaplan \& Broekaert, 2003). Further, FH explicitly creates an opportunity for mothers to re-learn parenting skills, develop positive relationships with their children, to acquire a productive work ethic, manage a stable household, and importantly to develop supportive links with the community (Fresh Hope, 2005; Sacks et al, 2004). Residents also take part in several communal/social activities in the facility (e.g., Sunday Roast...etc.). Residents also take part in general counseling sessions, education and training workshops.

Importantly, FH uses Graduates (i.e., Program Completers) to model and showcase a drug-free lifestyle and to provide a pattern of being able to live drug-free by demonstrating it in their day-to-day activities, work and pursuits. FH is similar to that of Daytop Village, as FH Graduates (i.e. who are staff) act as role models of living a drug-free life and are involved in the community as therapeutic agents (Kaplan \& Broekaert). This is also similar to the Oxford House Model, where the emphasis is on peer support for sobriety, shared responsibility and democratic leadership (Polcin \& Henderson 2008). To this end, a review of SLHs by Polcin and Henderson (2008) found that peer supported cultures and drug-free environments are likely to: foster change, make important mental health improvements and lead to a drug-alcohol free life. FH uses: relationships (e.g. between peers/residents, staff and residents and children and residents); language (e.g. residents strictly not allowed to talk about their drug history/habit or how they got into drugs/alcohol or their use or abuse with other residents), symbols; rituals; and purposeful relationship to build a sense of community around the individual. All activities are therapeutically orientated (i.e., relationship building, fostering positive attachment) and goal focused (e.g., learn to groom children properly) to develop a positive community around the resident.

\subsection{Purpose of Current Study}

The following study is a preliminary archival study (i.e., review of administrative files) of Fresh Hope over an eleven year period from 2000 to December 2011. This study reports only on preliminary descriptive findings on mothers, culture of FH against SCT constructs and not on children.

\section{Method}

\subsection{Participant Characteristics}

Residents are referred through Court, Hospitals, Drug Rehabilitation Units, Child Safety and some are Self-Referrals. Since 2000 to 2011 over 280 referrals were made to FH and of these only 154 were able to enter the program i.e. often due to the lack of beds. Ten residents were excluded from the facility as they compromised the safety and therapeutic process of other residents. The average age of residents $(\mathrm{N}=144)$ was 30 with an age range of 19 to 45 years among residents. The age of the children ranged from being born in the facility to 12 years of age. Preliminary demographical data (e.g., age, relationships status, drug use, medical concerns, psychological concerns, psychiatric concerns, and court orders....etc.). is collected at the time of admission into the facility. All participants consent to sharing their data for research purposes, and to inform effective running of FH. Over 15 graduates of FH were either staff or volunteers at FH and over 6 were living away from FH.

Table 1. Marital status of residents

\begin{tabular}{lcc}
\hline Type of relationships & $\mathrm{N}=144(\%)$ & Age M (range) \\
\hline Single & $133(92 \%)$ & $29(19-40)$ \\
Married & $8(.05 \%)$ & $35(25-41)$ \\
Divorced & $3(.02 \%)$ & $39(35-45)$ \\
\hline
\end{tabular}

Majority of the residents were single, however some residents might have been in a relationship prior to being a resident at Fresh Hope.

Table 2. Drug of use among residents

\begin{tabular}{lc}
\hline Name of Drug & Use $(\mathrm{N}=144)$ \\
\hline Amphetamines (e.g., Speed, Ice...Etc.) & $90(62 \%)$ \\
Alcohol & $57(39 \%)$ \\
Heroin & $27(18 \%)$ \\
Marijuana & $21(14 \%)$ \\
Morphine & $3(2 \%)$ \\
Nicotine & $140(97 \%)$ \\
\hline
\end{tabular}

Nicotine, Amphetamines and Alcohol appeared to preferred choice of drug among residents. No clinical assessments were conducted. 
Table 3. Presenting medical concerns of residents at the time of admission

\begin{tabular}{lc}
\hline Medical Concern & Number of residents \\
\hline Back Injury & 4 \\
Brain Damage & 1 \\
Cancer & 1 \\
Heart Condition & 1 \\
Hepatitis C & 16 \\
Lung Disease & 1 \\
\hline
\end{tabular}

Majority of the residents reported of being medically well at the time of the admission into the facility. No clinical assessments were conducted.

Table 4. Presenting psychological concerns of residents at the time of admission into the facility

\begin{tabular}{lc}
\hline Psychological Concerns & Number of residents \\
\hline Depression & 30 \\
Anxiety & 33 \\
Phobias & 10 \\
ADHD & 1 \\
\hline
\end{tabular}

Depression and anxiety were the two common psychological concerns that were reported by residents at the time of admission. No clinical assessments were conducted.

Table 5. Presenting psychiatric concerns of residents at the time of admission into the facility

\begin{tabular}{lc}
\hline Psychiatric Concerns & Number of residents \\
\hline Bi Polar & 5 \\
Borderline Personality Disorder & 3 \\
Post-Traumatic & 1 \\
Stress & 1 \\
Schizophrenia & 1 \\
\hline
\end{tabular}

Residents reported that the above Psychiatric concerns were diagnosed by a specialist prior to them coming to Fresh Hope. No clinical assessments were conducted.

Table 6. Presenting court order concerns of residents at the time of admission into the facility

\begin{tabular}{lc}
\hline Court Order Concerns & Number of residents \\
\hline Child Protection & 19 \\
Court Order & 3 \\
\hline
\end{tabular}

Child protection is a common legal concern among residents.

\subsection{Research Design}

This was a non-experimental archival study; wherein administrative files for the last 11years were reviewed against program completion by residents and whether they were living drug-free upon graduation (Broekaert, 2003).

\subsection{Instrument/s}

Treatment effect in post-release was simply measured by Graduates living drug free with or without continued participation in the community of FH either as staff, volunteers or visitors.

Treatment effect during the program in terms of living drug/alcohol free is quantitatively measured through random drug and alcohol urine tests alongside two in-house self-developed measures: one, a self-developed checklist was used to examine how many residents successfully completed all stages of the program (e.g. number of residents completed Stage 1, number of residents completed Stage 2, number of residents completed Stage 4 and number of residents completed Stage 4); and two, a self-developed SCT worksheet was used to map the culture of Fresh Hope during residency by looking at occurrences of positive negative behaviors (i.e. 12 questions), development of individual self (i.e. 9 questions) and social and community contributions (i.e. 9 questions). These questions were primarily developed to record institutional behaviors for residents. These were 
non-clinical in-house measures.

\subsection{Data Collection}

Data collected in FH is not clinical in nature. Data used in this study is in-house administrative record keeping archival data on behaviors (i.e. positive and negative) during residency and completion of the stages and the program.

\subsection{Data Analysis}

Treatment effect of post-release is presented in this paper is on number of residents living drug-free and those who have been in contact with FH after graduation. Treatment effects during the program for Personal/Community Engagement Experience/Program and through Community Drug Rehabilitative Treatment Experience/Program are reported measured against behaviors (i.e. positive and negative) and occurrences of activities during residency under behavior, individual and social/community in line with SCT constructs.

\section{Results}

A eleven year follow up in terms of Community Drug Rehabilitative Treatment Experience/Program results show that, over 49\% successfully completed Stage 1 Detoxification Phase, while 25\% completed Stage 2 Developing Positive Relationships and only about 15\% completed the full program and to date $90 \%$ remain drug-free. Post Release drug-free living ranged from 1 year to 12 years with a mean of 7 years. A significant finding is that $90 \%$ residents who graduate from Fresh Hope program were drug free and were also drug-free during their time in the facility measured primarily through random urine tests. All past graduates currently are either staff, visitors or volunteers at Fresh Hope.

Table 7. Behavioral outcomes during residency in Fresh Hope in terms of number of times an event occurred

\begin{tabular}{lc}
\hline Behavioral & No of times \\
\hline Drug/Alcohol free (i.e. Measured through & 0 \\
Random Urine test) & 0 \\
Assault & 0 \\
Property Damage & 0 \\
Theft & 0 \\
Suicide & 0 \\
Emergency calls to Police & 0 \\
Drug Overdose & 0 \\
Self Harming & 0 \\
Possession of contraband & 0 \\
Behaviour Misconduct & 0 \\
False Prescriptions & 0 \\
Verbal Violence & \\
\hline
\end{tabular}

At the behaviour level, FH appears to provide a safe, stable and constructive environment for residents.

Table 8. Individual outcomes during residency in Fresh Hope in terms of number of times an event occurred

\begin{tabular}{lc}
\hline Individual & No of times \\
\hline Positive/Structured mother/child interaction & 4 hours each day \\
Number of family reunions & $80 \%$ \\
Positive Family Mediations & $60 \%$ \\
Contact with husbands/partners & $90 \%$ \\
Contact with relatives & $95 \%$ \\
Custody of Children & $85 \%$ \\
Completing of Family Court Order & $90 \%$ \\
Marriages & 4 \\
Births & 7 \\
\hline
\end{tabular}

At an individual level, FH appears to provide positive opportunities toward residents developmental in terms of personal development and in developing relationships with others. 
Table 9. Social/Community outcomes during residency in Fresh Hope in terms of number of times an event occurred

\begin{tabular}{lc}
\hline Social/Community & Participation \\
\hline Completion of Community Service Orders & $80 \%$ \\
Volunteer & $100 \%$ \\
Education (P-12 and Higher Education) & $100 \%$ \\
Training (e.g. Cooking) & $100 \%$ \\
Employment & $100 \%$ \\
Community/Church Engagement & $100 \%$ \\
Treatment Participation & $100 \%$ \\
Financial contribution to School Chaplain & \$2000 (AUD) \\
Community Donations Annually & Host Fee BBQs \\
\hline
\end{tabular}

At a social/community level, FH appears to create a pro-social and altruistic community away from a deficit concept of community.

Anecdotal reports documented by Senior Counselor, Residents and House Parents, also report that all mothers appeared to have re-established positive relationships with their children, reconciled with their wider families and are active members in the community. This is further evident primarily through the amount of quality and productive time they are able to spend with their own children and the wider community. Mothers on average spent over $50 \%$ ( 8 hours per day) of their time with the children. Mothers report (i.e. mainly in informal discussions) a sense of achievement with increased positive self-talk and has having positive relationships with their children and a sense of belonging in the community.

\section{Discussion and Conclusion}

The current study confirms that, those residents who completed the program were drug-free during their time in Fresh Hope and in post-release $90 \%$ are still drug-free and active and productive participants in the community. This study confirms that therapeutic communities operating under the premise of a democratic method of treatment provide a useful non-instructive alternative to clinical treatments (De Leon, 1993; Mills \& Harrison, 2007; NDS; Provert, 2006; Vaughan, 2004; Wolfman, 2009; Zweben, 1993). FH addressing addictive behaviors at the individual, behavior and environment level seems to have sustained a positive long-term change (Bandura, 1986). This finding is similar to Sacks et al. (2004) and Lees et al. (2004) who found a positive effect for treatment through community such as FH. While a great majority completed Stage 1 of Detoxification, and only a small cohort completed the full program (i.e. 15\%); which is in line with De Leon (1993) who notes that “...most substance abusers do not complete planned duration of treatment....[and that]... most reliable predictor of successful outcome is retention in treatment..." (p.104; Toumbrourou et al., 1998). The current study also confirms the findings of Polcin and Henderson (2008) and Wolfman (2009) for low rates of treatment retention. However, could it be possible that, rather than the kind of treatment that retention rates are more representative of the kind of client (e.g. high motivated) that comes to seek support and recovery through a therapeutic community. To this Provert (2006) notes that “...fundamentally clinical potency within the TC is maximized when its members achieve intrinsic motivation..." (p. 34). Perhaps then one way to increase retention rates is to explicitly address the intrinsic motivation in clients seeking assistance.

Even though the current study has found 15\% to complete the program and now to remain drug free, it could be possible that these individuals were motivated to give up drugs even before they came to FH. Perhaps in a way they were already reformed in a sense and began their rehabilitative journey, where coming to FH could have been a preferred choice of convenience (De Leon, 1993). Further not knowing the level of need (e.g., high, medium or drug) among residents in terms of their addiction prior to coming to $\mathrm{FH}$, it is difficult to interpret the results, and caution must be exercised in terms of the generalizability of these results. In addition, given that FH does not offer a clinically based drug-rehabilitative program, the question of 'how' does therapeutic community actually lead to breaking the cycle of addiction is still not clear from a psycho-medical model (Lees et al., 2004). Such unanswered questions can lead to the assertion that therapeutic communities are nothing more than mere social rehabilitation, with very few clinically measured again or outcomes for the long-term sustainable change (Wolfman, 2009). While such criticisms are valid and necessary, perhaps one way to measure the effectiveness is to compare FH's outcomes to a control group and/or a clinical center.

While a number of participants reported of medical, psychiatric and/or psychological concerns to the Psychiatrist, it was difficult to gauge and measure positive/qualitative gains as these reports were confidential, but all mothers 
fully participated in all activities and appeared to have adhered to the therapeutic culture of FH. Even though mental health concerns were noted for participants at the time of the induction, it was difficult to gauge how each of them benefited from being in the facility as FH does not have pre and post measures until recently. Hence, more research is needed to review residents medical, drug and psychiatric needs and concerns against qualitative gains, so as to explore to what extent $\mathrm{FH}$ as a therapeutic community creates a positive sustainable change in terms of mental health and well-being of the residents. However, in terms of qualitative gains this study has confirmed the findings of De Leon (1993) that there is an increased positive self-talk and improvements in psychological (e.g. less likely to anxious or agitated) wellbeing among residents.

Importantly, outcomes on behaviors, individual and social/community against SCT was useful at it confirmed FH to be a positive pro-social therapeutic community. Further, such outcomes also are indicative of residents experiencing a positive sense of self and for them to develop the much needed constructive core beliefs by exposing them to altruistic and positive social behavior, consequently safeguarding them from anti-social behaviors, drug-culture, crime and criminality. In this FH provides an environment for change to occur as it minimizes disruptions of lives (e.g., especially between mothers and their children), increases social connectedness, advances adult personal development, reduces personal treatment costs (when compared to conventional medical treatments) and importantly provides a less intensive treatment environment (Cardoso et al., 2003; Provert, 2006).

While democratic therapeutic communities provide non-intrusive therapeutic support, there is a great need for such communities to collect quality and quantitative data in an objective manner. Data should be collected accurately, reliably and should be properly manage in terms of showing treatment progress at each stage qualitatively (e.g. developing meaningful relationships) and quantitatively (e.g. completed all tasks associated with Stage 1, and now aware of Drug Withdrawal symptoms). The advantage of treatment in therapeutic communities is the rich data that it is able to collect from antidotal to formal data on different on residents (i.e. behavior, parenting....ect) through different lens (i.e. peers, staff) which is likely to provide a unique insight into how therapeutic communities precisely come to make a difference in the lives of those dealing with difficult life issues.

\section{References}

Andrews, D. A., \& Bonta, J. (1994). The psychology of criminal conduct. Cincinnati: Anderson Publishing Co.

Bandura, A. (1986). Social Foundations of Thought and Action: A Social Cognitive Theory Englewood Cliffs, N.J: Prentice-Hall.

Bandura, A. (2003). Bandura's Social Cognitive Theory [videorecording]: An introduction. San Luis Obispo, CA: Davidson Films.

Broekaert, C. K. (2003). An introduction to research on the social impact of the therapeutic community for addiction. Journal of International Social Welfare, 12, 204-210.

Cardoso, D. S. E., Chan, F., Berven, L. N., \& Thomas, R. K. (2003). Measuring readiness to change among individuals in residential therapeutic community programs for treatment of substance abuse. Rehabilitation Counselling Bulletin, 47(1), 34-44.

Clinton, R. H. (1996). It takes a village to raise a child, and other lessons children teach us. New York: Simon \& Schuster.

Collins, D., \& Lapsley, H. (2008). The costs of tobacco, alcohol and illicit drug abuse to Australian society in 2004-05. National Drug Strategy monograph series no. 66. Canberra: Australian Government Department of Health and Ageing.

De Leon, G. (1986). The therapeutic community for substance abuse: perspective and approach. In G. De Leon \& J. T. Ziegenfuss. Jr. (Eds.).Therapeutic Communities for Addictions. Charles C. Thomas: Springfield, IL.

De Leon, G. (1989). Therapeutic communities for substance abuse: Overview of approach and effectiveness. Psychology of Addictive Behaviours, 3(3), 140147.

De Leon, G. (1993). What psychologists can learn from addiction treatment research. Psychology of Addictive Behaviours, 7(2), 103-109.

De Leon, G. (2000).The Therapeutic Community: Theory, Model and Method. New York: Springer Publishing.

De Leon, G., \& Jainchill, J. (1982). Male and female drug abusers: Social and psychological status 2 year after treatment in a therapeutic community. American Journal of Drug Alcohol Abuse, 8(4), 465-497. 
De Leon, G., Sacks, S., Staines, G., \& McKendrick, K. (2000). Modified therapeutic community for homeless MICAs: Treatment outcomes. American Journal of Drug and Alcohol Abuse, 26(3), 461-480.

Fresh Hope. (2005). Fresh Hope Policy and Procedures. Fresh Hope Inc.

Gottdiener, H. W. (2001). The utility of individual supportive psychodynamic psychotherapy for substance abusers in a therapeutic community. The Journal of The American Academy of Psychoanalysis, 29(3), 469-481

Gottfredson, R. M., \& Hirschi, T. (1990). A General Theory of Crime. California: Stanford University Press.

Huffman, K. (2004). Psychology in Action. ( $7^{\text {th }}$ Ed.). John Wiley \& Son Inc: NJ

J-F. (2011). Conceptualization of Treatment Logic: Faith Base Unit (FBU): Rimutaka Prison, New Zealand Corrections. A Response to FBU Evaluation by Department of Corrections, New Zealand (Published September 2010).

Jones, M. (1959).Towards a clarification of the 'therapeutic community' concept. The British Journal of Medical Psychology, 32(3), 200-205.

Kaplan, C., \& Broekaert, E. (2003). An introduction to research on the social impact of therapeutic community for addiction. International Social Welfare, 12, 204-210.

Kennard, D. (2004). The Therapeutic Community as an adaptable treatment modality across different setting. Psychiatric Quarterly, 75(3), 295-307.

Lees, J., Manning, N., \& Rawlings, B. (2004). A culture of enquiry: Research evidence and the therapeutic community. Psychiatric Quarterly, 75(3), 279-294.

Melnick, G., \& De Leon, G. (1999). Clarifying the nature of therapeutic community treatment: The survey of essential elements Questionnaire (SEEQ). Journal of Substance Abuse Treatment, 16, 307-313.

Mills, A. J., \& Harrison, T. (2007). John Rickman, Wilfred Ruprecht Bion, and the origins of the therapeutic community. History of Psychology, 10(1), 22-43.

Murray, J., Cheliotis, L., \& Maurna, S. (2007). Social Factors and Crime. Edited by Parker, M. Dynamic Security: The Democratic Therapeutic Community in Prison. London: Jessica Kingsley Publishers.

National Drug Strategy 2010-2015 (NDS). (2011). A framework for action on alcohol, tobacco and other drugs. Ministerial Council on Drug Strategy

National Drug Strategy Household Survey. (2011). Australian Institute of Health and Welfare. Canberra.

National Institute on Drug Abuse (NIDA). (2004). Therapeutic Community. NIDA Research Report Series. NIH Publication Number 02-4877. Rockville, MD: NIDA.

Nielsen, L. A., \& Scarpitti, R. F. (1997). Changing the behaviour of substance abusers: Factors influencing the effectiveness of therapeutic communities. Journal of drug Issues, 27(2), 279-294.

Perfas, B. F., \& Spross, S. (2007). Why the concept-based therapeutic community can no longer be called drug-free. Journal of Psychoactive Drugs, 39(1), 69-79.

Polcin, L. D., \& Henderson, M. D. (2008). A clean and sober place to live: Philosophy, structure and purported therapeutic factors in sober living houses. Journal of Psychoactive Drugs, 40(2), 153-159.

Provert, P. (2006). Clues to the future of the therapeutic community. Addiction Professional, 4(7), 33-36.

Rapoport, R. N. (1960). Community as Doctor. London: Tavistock.

Sacks, S., Sacks, J. Y., Bernhardt, A. L., Harle, M., \& De Leon, G. (1997). Homelessness prevention TC for addicted mothers: Program Manual. Rockville, MD: Center for Mental Health Services (CMHS)/Centre for Substance Abuse Treatment (CSAT).

Sacks, S., Sacks, J. Y., McKendrick, K., Pearson, F. S., Banks, S., \& Harle, M. (2004). Outcomes from a therapeutic Community for homeless addicted mothers and their children. Administration and Policy in Mental Health, 31, 4.

Safran, D. J., \& Muran, J. C. (1998). The therapeutic alliance in brief psychotherapy: General principles. In J. D. Safran, \& C. J. Muran. The therapeutic alliance in brief psychotherapy. Washington: American Psychological Association.

Smith, S. (2011). Therapeutic Community: A century at Gould Farm. Communities, 150, 46-51. 
Sutherland, E. H. (1947). Principles of Criminology. (4th Ed). Philadelphia: J.B. Lippincott.

Toumbrourou, W. J., Hamilton, M., \& Fallon, B. (1998). Treatment level progress and time spent in treatment in the prediction of outcomes following drug-free therapeutic community treatment. Addictions, 9(7), 1051-1064.

Vaughan, F. R. (2004). Therapeutic Communities: A therapeutic bridge. Journal of Psychoactive Drugs, 36(2), 265-271.

Ward, A. (2003). The Core Framework. In A. Ward., Kasinski, K., Pooley, J., \& Worthington (Ed.). Therapeutic communities for children and young people: Therapeutic communities 10. London: Jessica Kingsley Publishers.

Wolfman, N. J. (2009). No man is an Island: Social predictors in treatment retention in a therapeutic community. Doctor of Philosophy Thesis. Department of Psychology. University of Maryland.

Yates, R., \& Wilson, J. (2001). The modern therapeutic community: Dual diagnosis and the process of change: In B. Rawlings \& R. Yates (Eds.). Therapeutic Communities for the Treatment of Drug Users. London: Jessica Kingsley Publishers.

Zweben, E. J. (1993). Recovery oriented psychotherapy: A model for addiction treatment. Psychotherapy, 30(2), $259-268$.

\section{$(\mathrm{cc}) \mathrm{BY}$}

This work is licensed under a Creative Commons Attribution 3.0 License. 